\title{
Sub-barrier fusion of the oxygen isotopes: A more complete picture
}

\author{
J. Thomas, Y. T. Chen, S. Hinds, ${ }^{*}$ D. Meredith, and M. Olson \\ Division of Physics, Mathematics, and Astronomy, California Institute of Technology, Pasadena, California 91125
}

(Received 24 December 1985)

\begin{abstract}
We have measured the sub-barrier fusion cross sections for ${ }^{16,17,18} \mathrm{O}$ on ${ }^{16} \mathrm{O}$ and we present the data and the experimental method in detail. The data were analyzed with a one-dimensional potential barrier inversion model, a two-dimensional incoming wave boundary condition model, and a two-dimensional Wentzel-Kramers-Brillouin model. We find that a multidimensional model is necessary to describe the enhanced fusion cross sections in oxygen and the two-dimensional incoming wave boundary condition model suggests that a complete description of the fusion, inelastic, and elastic cross sections is possible.
\end{abstract}

\section{INTRODUCTION}

Balantekin et al. ${ }^{1}$ have demonstrated that the enhancement of sub-barrier fusion cross sections is not a result of improperly chosen parameters in a simple onedimensional model describing the fusion process. They used a simple model, based on the Wentzel-KramersBrillouin (WKB) approximation, to invert the fusion cross sections to yield an effective one-dimensional $S$ wave potential. For many low mass systems ${ }^{2}$, the model provided a very reasonable potential and so an effort has been made to describe the fusion of all the $p$ shell nuclei with one universal proximity potential. ${ }^{3}$ The program was modestly successful but there were also some conspicuous inconsistencies in the analysis of ${ }^{16} \mathrm{O}+{ }^{16} \mathrm{O},{ }^{13} \mathrm{C}+{ }^{16} \mathrm{O}$, and ${ }^{12} \mathrm{C}+{ }^{13} \mathrm{C}$ and it is not clear whether this is due to experimental errors or due to a deeper physical mechanism. The latter possibility was suggested by the unusual backbending potentials derived from the $\mathrm{Ni}+\mathrm{Ni}$ and $\mathrm{Ni}+\mathrm{Ge}$ sub-barrier fusion data of Beckerman et al. ${ }^{4,5}$ In these heavy systems, it is possible that the structure of the nuclei or the nuclear surfaces play a role in the fusion process.

With these results in mind, we have studied the fusion of the oxygen isotopes ${ }^{16,17,18} \mathrm{O}$ with ${ }^{16} \mathrm{O}$. These nuclei offer the opportunity to study fusion below the Coulomb barrier in a systematic manner where the structure and reactions of the nuclei are well understood.

Our working hypothesis was that sub-barrier fusion is a multidimensional process where neutron transfer and inelastic channels couple to the fusion channel and might produce an enhanced fusion cross section. We will show later that a simple model described by Landowne, Dasso, and Winther ${ }^{6,7}$ provides a good description of the data, and that coupling to the inelastic channel is the dominant contribution to the sub-barrier fusion process in oxygen, since neutron transfer reactions were not observed in our experiments.

In section II we describe the experimental procedures in detail before describing the data analysis in section III and presenting our data in section IV. In section $\mathrm{V}$ we discuss the need for a multidimensional model, and show that a two-dimensional model is a satisfactory approximation to describe the fusion and inelastic excitation of ${ }^{16} \mathrm{O}+{ }^{16} \mathrm{O}$, ${ }^{16} \mathrm{O}+{ }^{17} \mathrm{O}$, and ${ }^{16} \mathrm{O}+{ }^{18} \mathrm{O}$.

\section{EXPERIMENTAL METHOD}

The data were collected at the Caltech-ONR EN tandem Van de Graaff accelerator facility. Beam energies ranged from approximately $7 \mathrm{MeV}$ to $12.5 \mathrm{MeV}$ in the center of mass for each of the systems studied. The data were collected in $0.25 \mathrm{MeV}$ steps in order to be sensitive to any possible oscillations in the cross section with spacing similar to those which have been seen in the ${ }^{12} \mathrm{C}+{ }^{12} \mathrm{C}$ cross section ${ }^{8}$. The rare oxygen beams were generated with a Duo-plasmitron ion source by introducing isotopically enriched water ${ }^{9}$ vapor directly into the source. (We found it necessary to gold plate the interior of the source and plasma cone in order to be able to extract usable yields of the ${ }^{17} \mathrm{O}$ and ${ }^{18} \mathrm{O}$ beams, otherwise the ${ }^{16} \mathrm{O}$ yield from the oxide on the interior of the source masked the rare beams.)

The $4^{+}$charge state was selected for the beam and intensities on target ranged from $50 \mathrm{nA}$ at the highest energies to $4 \mu \mathrm{A}$ at the lowest. The targets were separated from the accelerator vacuum by an in line cold trap and a differential pumping system. This pumping section was also an ideal point to locate a $5 \mathrm{~mm}$ diameter aperture for defining the shape of the beam. The aperture was electrically insulated so that the beam could be maximized passing through it, and the halo was usually less than $1 \%$ of the total beam. An in-line ion pump helped to ensure that ultra-high vacuum conditions were maintained in the vicinity of the target. For the ${ }^{16} \mathrm{O}$ and ${ }^{18} \mathrm{O}$ work, the vacuum with beam on target was typically $1 \times 10^{-8}$ Torr, and for the ${ }^{17} \mathrm{O}$ work the vacuum was typically $1 \times 10^{-9}$ Torr. The quality of the vacuum at the target is vitally important because of the possibility of carbon buildup since carbon-oxygen fusion has a lower threshold and could easily dominate the derived fusion yield ${ }^{10}$. The problem of carbon buildup is aggravated by the necessity of cooling the target in order to prevent its destruction.

The target was mounted on a special water cooled conflat flange shown in figure 1 . It was essentially a modi- 


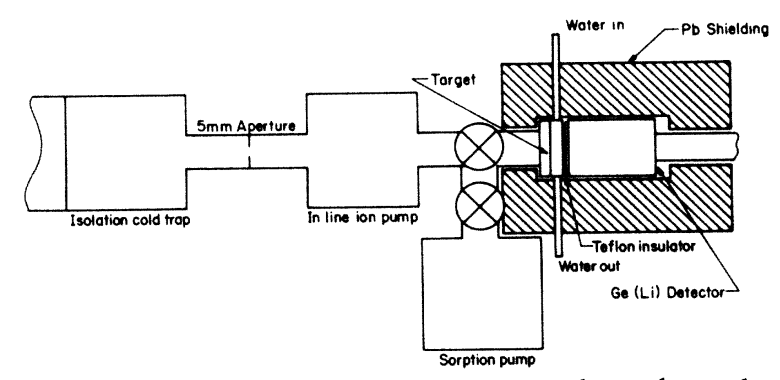

FIG. 1. The $\mathrm{Ta}_{2} \mathrm{O}_{5}$ target was mounted on the end of an ultra-high vacuum beam line and was water cooled to prevent degradation of the oxide layer. Typical vacuum conditions near the target were $10^{-8}$ to $10^{-9}$ Torr.

fied blankoff that was bored out to allow cooling water to come in contact with the target and also to provide as thin a window as possible to reduce $\gamma$ ray attenuation. The target was the same diameter as the copper gasket for the flange and was placed between the knife edge and the copper gasket. This seal showed no signs of leaking, and the quality of the vacuum was dominated by the cleanliness of the parts before assembly. We experimented with air cooling and a closed loop of deionized water with a recirculating pump. Both systems had to be abandoned because they generated an excessive amount of microphonics in the germanium detector which was in contact with the target mount. Instead, we used a one pass siphon of water to cool the target. The total amount of leakage current was below $1 \mathrm{nA}$ with this system. The target and cooling system were electrically insulated from the accelerator for beam current integration and magnetic suppression of the secondary electrons was used. The charge integration was checked by observing the Coulomb excitation of a blank tantalum target and the two measures agreed to better than $1 \%$.

The detector was mounted as close to the target as possible but this distance was limited by the thickness of the target cooling system. The detector was mounted about 5 $\mathrm{mm}$ behind the target and was separated from it by a Teflon insulator and $1.5 \mathrm{~mm}$ of $\mathrm{Pb}$ to stop low energy $\mathrm{x}$ rays. The detector was a $100 \mathrm{~cm}^{3} \mathrm{Ge}(\mathrm{Li})$ detector with a resolution of $2 \mathrm{keV}$. The detector resolution was not particularly important because most of the observed $\gamma$ rays were Doppler shifted. The Doppler shifting is a result of the nuclear levels of interest having lifetimes of a few picoseconds or less which are comparable to the stopping time of the ions in the target backing. The Doppler shift was as large as $4 \%$.

The detector was shielded with $10 \mathrm{~cm}$ of lead in all directions. Its photopeak efficiency was determined with a mixed Eu-Sm source that was calibrated by the National Bureau of Standards ${ }^{11}$. The total efficiency was determined with a ${ }^{22} \mathrm{Na}$, a ${ }^{137} \mathrm{Cs}$, and a ${ }^{60} \mathrm{Co}$ source. These sources were secondary standards that were ultimately traceable back to the NBS. The efficiency of the detector was measured with the source in the same position as the target, with water in the cooling jacket and all other conditions similar to those when the data were taken.
Dead time was monitored by triggering a pulser with the output of the beam current integrator. The pulser was applied to the test input of the detector and so the total live current was recorded with each spectrum. The dead time was kept below $10 \%$ at all times. The gamma ray yields were recorded with a Tracor-Northern model 7200 multichannel analyzer with 2000 channel resolution. The analyzer spanned a range from 0 to $4 \mathrm{MeV}$ above which the detector photoefficiency dropped so low that it was not worthwhile to collect data.

The targets were made of anodized tantalum sheets and were compatible with other ultra-high vacuum materials. The anodized layers were relatively thin so that corrections for the finite thickness of the target could be done without the necessity of subtracting thick target yields. We tried several other oxides; the most promising was $\mathrm{WO}_{3}$, but we found that the stoichiometry of the oxide was not stable when bombarded by the beam. The $\mathrm{Ta}_{2} \mathrm{O}_{5}$ layer targets were stable under beam bombardment and could be made to any desired thickness. Initially, the $\mathrm{Ta}$ disk was stripped clean with a mixture of acids ${ }^{12}$. This step removed any contamination on the surface of the Ta. The targets were anodized in a solution of $0.01 N \mathrm{KI}$ (Ref. 13) with an anodizing current of $15 \mathrm{~mA}$. Although the target thickness is proportional to the voltage applied, we found it more convenient to integrate the total charge passing through the cell. A simple calculation yielded the target thickness if it was assumed that the anodizing procedure was $100 \%$ efficient. In fact, we found that the procedure was about $95 \%$ efficient. During the anodizing procedure, the target changes color several times and we found that we could estimate the target thickness to within $5 \%$ by noting the color. We typically made targets with a $100 \mu \mathrm{g} \mathrm{Ta}_{2} \mathrm{O}_{5}$ layer. The oxide has a high sputtering yield and so the target could degrade when bombarded by the beam. The cure for this problem was to evaporate $200 \mu \mathrm{g}$ of gold onto each target which was sufficient to retain the low energy sputtered ions ${ }^{14}$.

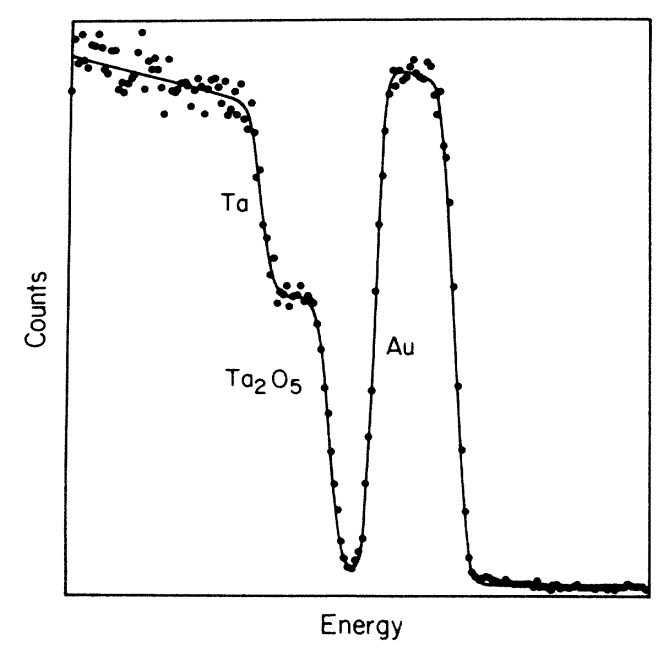

FIG. 2. A typical Rutherford backscattering spectrum of ${ }^{16} \mathrm{O}$ ions from an ${ }^{16} \mathrm{O}$ target. The $\mathrm{Au}$ anti-sputtering layer can be used to determine the energy loss of ${ }^{16} \mathrm{O}$ ions in this layer and in the $\mathrm{Ta}_{2} \mathrm{O}_{5}$. The oxide layer shows up as a step in the Ta peak. 

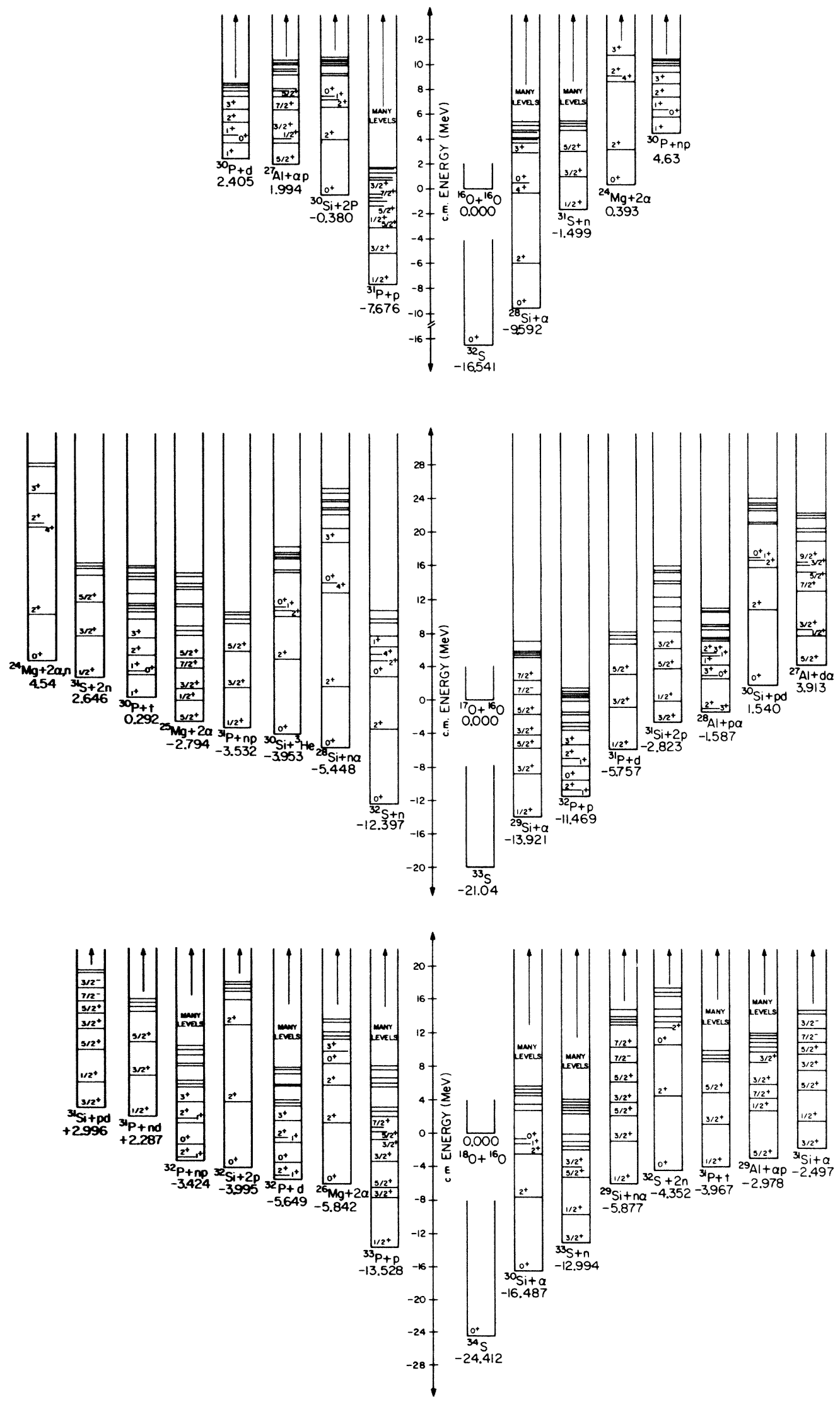

FIG. 3. $Q$ value diagrams for ${ }^{16} \mathrm{O}+{ }^{16} \mathrm{O},{ }^{16} \mathrm{O}+{ }^{17} \mathrm{O}$, and ${ }^{16} \mathrm{O}+{ }^{18} \mathrm{O}$ showing the energetically allowed exit channels for the decay of the various compound systems. 
We routinely measured the gamma ray yield at $24 \mathrm{MeV}$ $\left(E_{l a b}\right)$ bombarding energy in order to check on the deterioration of the target. The check was performed every $15 \mathrm{mC}$ and usually indicated no loss. The maximum deterioration of any target was a $15 \%$ decrease in thickness of one target after $150 \mathrm{mC}$ of beam bombardment. All of the data were corrected for the observed target thickness variations. These runs at $24 \mathrm{MeV}$ were also useful for checking the rate of carbon buildup on the targets as will be described later.

The target thickness was checked by Rutherford backscattering of ${ }^{16} \mathrm{O}$ ions at $20 \mathrm{MeV}\left(E_{\text {lab }}\right)$. Figure 2 shows a typical RBS spectrum collected at 160 degrees. The gold peak stands alone and the thickness of this layer and the energy loss $(d E / d x)$ can be estimated from the FWHM and the total number of counts in this peak. In fact, we found that the measured $d E / d x$ is $15 \%$ less than the values found in the tables of Northcliffe and Schilling ${ }^{15}$. Therefore in estimating the thickness of the $\mathrm{Ta}_{2} \mathrm{O}_{5}$ layer, we reduced the $\mathrm{Ta} d E / d x$ by $15 \%$ (both $\mathrm{Ta}$ and $\mathrm{Au}$ are based on the same extrapolation). The oxygen peak is not directly observable in figure 2 but the effect of the oxygen on the surface of the blank is to reduce the density of the $\mathrm{Ta}$ and this can clearly be seen as a step in the figure. The thickness of the targets was estimated from the energy lost in this step.

\section{ANALYSIS}

The fusion of oxygen nuclei leads to a highly excited compound nucleus which decays by particle and $\gamma$ ray emission into several well-defined exit channels. Some of the exit channels are excited above particle threshold and decay into yet a second set of exit channels. We considered all energetically allowed exit channels up to and including three-body final states (e.g., ${ }^{29} \mathrm{Si}+\alpha+\mathrm{n}$ ). These reaction channels are shown plotted as a function of excitation energy above the compound nuclear ground state in figure 3 for ${ }^{16} \mathrm{O}+{ }^{16} \mathrm{O},{ }^{16} \mathrm{O}+{ }^{17} \mathrm{O}$, and ${ }^{16} \mathrm{O}+{ }^{18} \mathrm{O}$. The density of states in each nucleus is suggested by the levels that are drawn for each heavy residual nucleus. The exit channels are dominated by the breakup into $\mathrm{p}, \mathrm{n}, \mathrm{d}, \mathrm{t},{ }^{3} \mathrm{He}$, and $\alpha$ particles.

The method of our analysis is somewhat different from the method traditionally used in sub-barrier fusion cross section measurements. Instead of detecting the heavy ion recoils, we detect the characteristic $\gamma$ rays coming from the de-excitation of the residual nuclei. Our techniques are similar to those pioneered by Spinka and Winkler ${ }^{8}$, and Christensen et al. ${ }^{10}$ In particular, Spinka and Winkler have made extensive measurements to verify that the cross sections obtained by the $\gamma$ ray techniques are the same as cross sections determined from heavy ion recoil measurements or light particle detection schemes.

In order to calculate fusion cross sections from the $\gamma$ ray yields, we had to estimate the probability of populating a particular level in a particular evaporation residue. We assumed that the Hauser-Feshbach (HF) statistical model formalism was satisfactory for this purpose ${ }^{16}$. The analysis did not require absolute cross sections and depended only on their relative magnitudes.
The partial fusion cross section was then derived from the $\gamma$ ray yield via

$$
\sigma \propto \operatorname{Yield}(\gamma, E) / \beta \epsilon\left(E_{\gamma}\right)
$$

where $\beta$ is the relative probability of populating a given level including the contributions from de-excitation of higher lying levels in the residual nucleus and $\epsilon\left(E_{\gamma}\right)$ is the detector photopeak efficiency discussed in section II. We therefore need only one $\gamma$ ray for each residual nucleus to calculate $\sigma$; more $\gamma$ rays make the deduced cross sections more reliable.

We used the computer code HAUSER*5 (Ref. 17) to calculate the relative probabilities, $\beta$. This is a convenient application of the statistical theory which includes a table of global optical model parameters for reactions with most of the light particles. The code required minor modifications in order to calculate the three-body cross sections. These calculations were done in two steps: in the reaction $W+X$ goes to $Y+a+b$, first the reaction $W+X$ goes to $Z+b$ was calculated where $Z$ is the compound system $(Y+a)$. This gives the cross section to each energetically accessible level in $Z$. Second, the cross section for the decay of $Z^{*}$ to $Y+a$ was calculated to yield the probability of the decay of any excited level in $Z$ to the residual nucleus $Y$. The cross section to each level in $Z$ was then weighted by these probabilities to yield the cross section to each level in $Y$.

HAUSER *5 requires the input of the mass, charge, and binding energy of each nucleus as well as the spin, parity, and energy of all of the known levels ${ }^{18}$ and an estimate of the level density ${ }^{19}$ at energies that have not been explored experimentally. The code then computes the cross section to each level. We normalize these cross sections to the total cross section to yield the relative values. Thus the calculations are insensitive to errors in the magnitude of the cross section and depend only on our relative knowledge of the optical model parameters.

The $\beta$ values are derived from the relative cross sections by allowing the excited states, below particle threshold, to decay to the ground state with the experimentally known branching ratios ${ }^{18}$. The $\beta$ factor calculations also take into account the probability of $\gamma$ ray summing into or out of the peak of interest ${ }^{20}$. The relative probabilities, $\beta$, vs $E_{c . m}$. are plotted in figure 4 for all of the $\gamma$ rays that were observed in these experiments.

The determination of the partial cross sections was straightforward, apart from a few minor complications. For example, the HF calculations for the three-body cross sections were unreliable when the $\beta$ values were very small. This manifested itself by predicting excessively large cross sections. The ${ }^{27} \mathrm{Al}+\alpha+\mathrm{n}$ channel in ${ }^{16} \mathrm{O}+{ }^{16} \mathrm{O}$ is the only important example of this problem. Here, the calculated $\beta$ factor is very small (see figure 4) and at low energies the inferred cross section would actually increase. Since this channel represents only about $10 \%$ of the total cross section, we have assumed that the $\beta$ value at 24 $\mathrm{MeV}\left(E_{l a b}\right)$ is correct and have normalized the HF cross section to this point. The rest of the ${ }^{27} \mathrm{Al}$ data were then assumed to follow the $\mathrm{HF}$ shape. For the ${ }^{16} \mathrm{O}+{ }^{18} \mathrm{O}$ fusion reaction, two channels were totally unobserved. Since these channels represent less than $1 \%$ of the total cross 
section, as estimated with HAUSER *5, we have also used the HF cross sections for these channels.

The $\gamma$ ray yields were estimated by assuming a linear background under each of the observed peaks. Typical spectra collected at $E_{\text {lab }}=24 \mathrm{MeV}$ are shown in figure 5 . This figure demonstrates that the lines are severely Doppler shifted; by as much as $4 \%$ in some cases. We made no attempt to calculate these line shapes and merely integrated the total area of the peak plus Doppler shifted part. The Doppler shifting did cause some ambiguities. The $2.23 \mathrm{MeV}$ peak in the ${ }^{16} \mathrm{O}+{ }^{16} \mathrm{O}$ spectrum has contributions from ${ }^{30} \mathrm{Si},{ }^{31} \mathrm{P},{ }^{31} \mathrm{~S}$, and other nuclei. Usually we avoided these peaks, but on occasion there was only one observed line for a particular exit channel and we had to use these mixed peaks. In these cases, we used the HAUSER $* 5$ cross sections to estimate the relative contribution of each nucleus to the observed yield, and the yield was then multiplied by this fraction.

The cross sections had to be corrected for the energy loss of the beam in the target. The energy loss in the Au anti-sputtering layer merely shifted the average beam energy, but the energy loss in the $\mathrm{Ta}_{2} \mathrm{O}_{5}$ layer was more complex. The cross section is a strong function of energy, so we expanded the cross section in a Taylor series to second order and estimated the first and second derivatives from the data. The calculated differential cross sec-
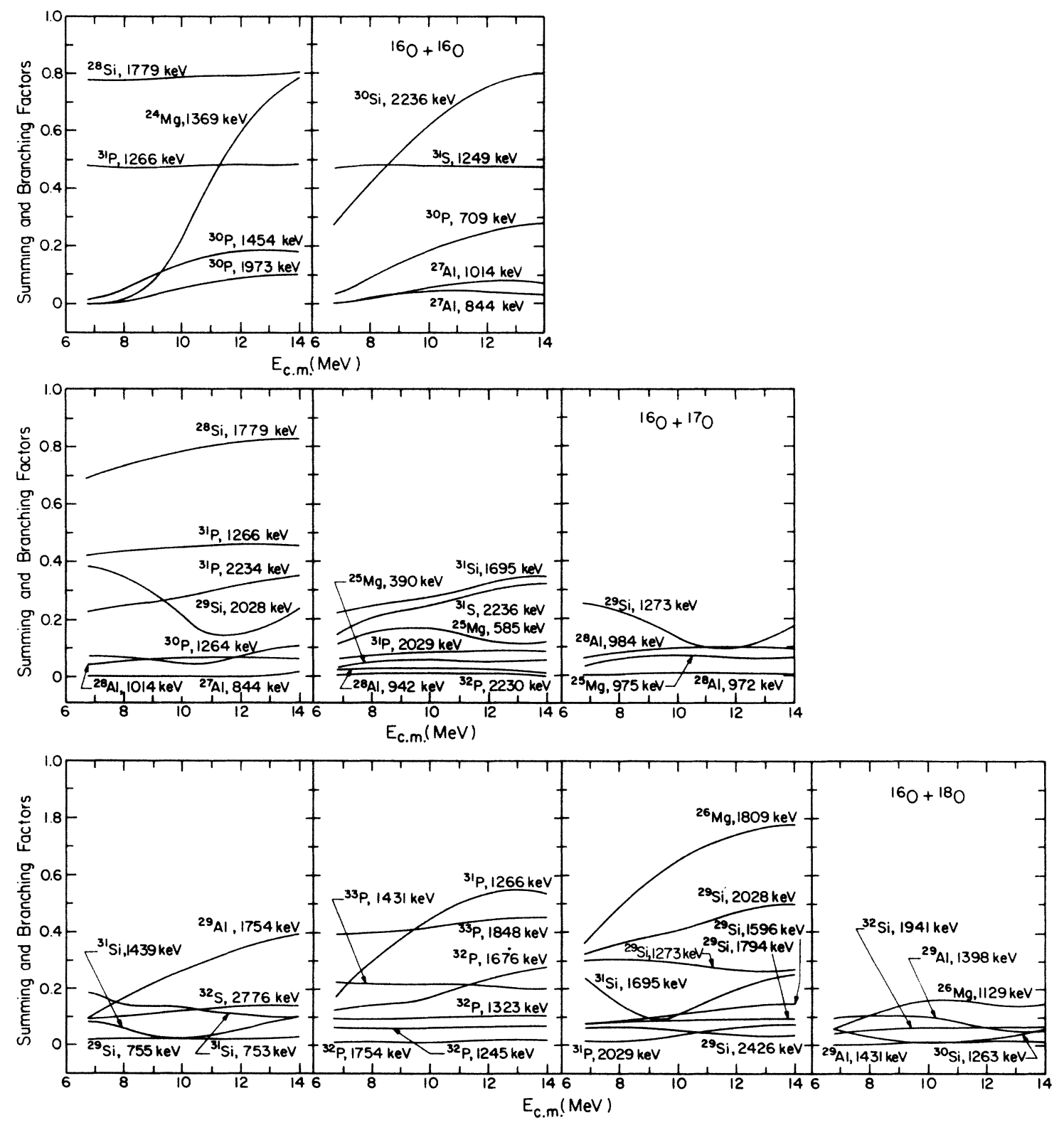

FIG. 4. The summing and branching factors, $\beta$, are plotted as a function of the center of mass energy for each of the systems studied. 

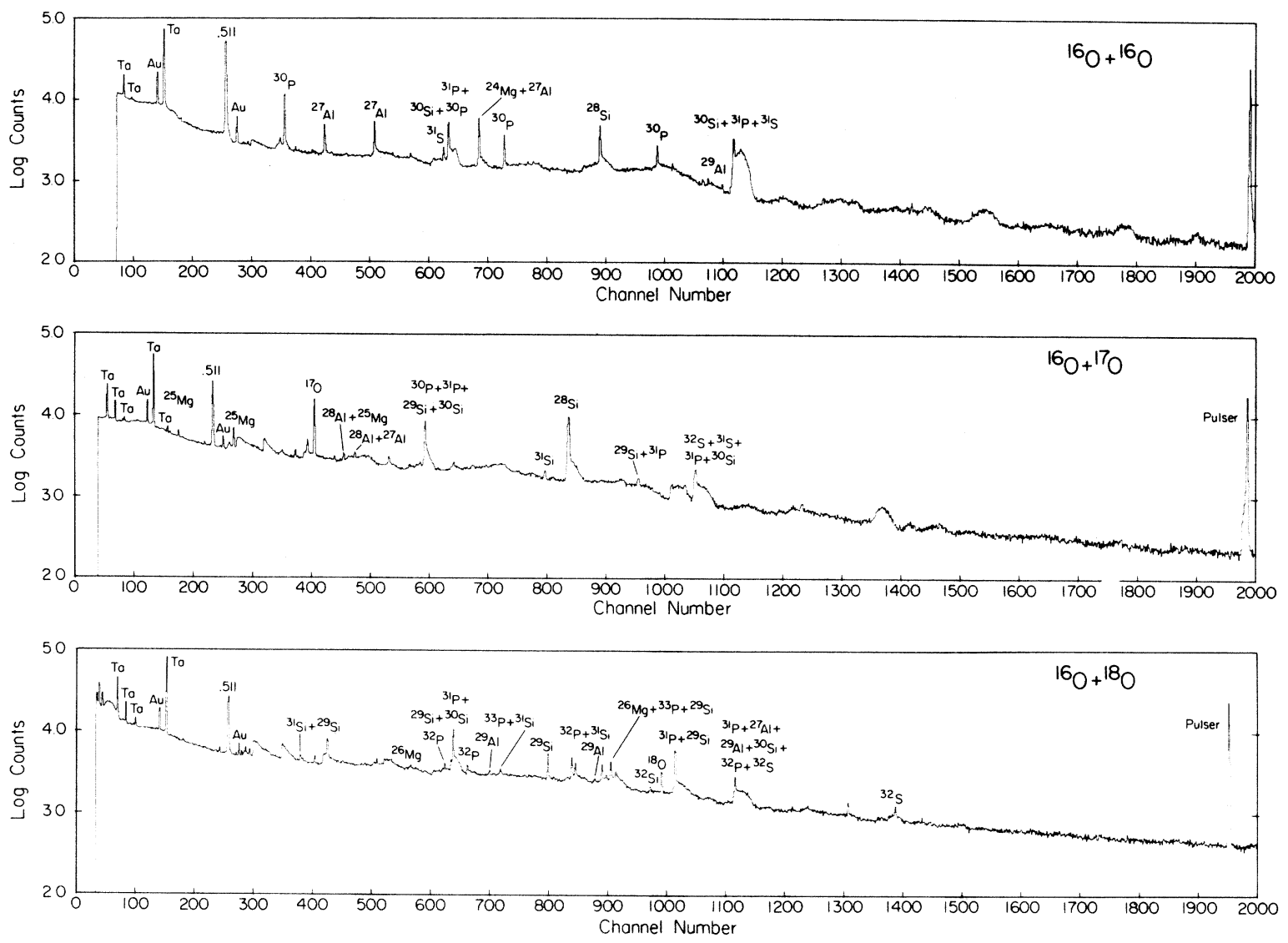

FIG. 5. Typical $\gamma$ ray spectra observed at $24 \mathrm{MeV}$ bombarding energy. The energy calibration is such that the $\gamma$ ray energy, in $\mathrm{MeV}$, is half of the channel number.

tion was then integrated over the energy interval of the beam in the target $\left(\sim 0.5 \mathrm{MeV}_{\text {lab }}\right)$ and the cross section at the surface of the target was estimated. Figures 6, 7, 8 plot these differential cross sections as a function of energy. Typically the observed yields at low energy were increased by $25 \%$ to account for the energy loss in the target.

\section{DATA}

Typical $\gamma$ ray spectra produced by ${ }^{16} \mathrm{O}+{ }^{16} \mathrm{O},{ }^{16} \mathrm{O}+{ }^{17} \mathrm{O}$, and ${ }^{16} \mathrm{O}+{ }^{18} \mathrm{O}$ fusion evaporation residues are shown in figure 5. These spectra were collected at $24 \mathrm{MeV}\left(\mathrm{E}_{l a b}\right)$ and each was one of the periodic runs used to monitor the target thickness. The energy calibration was chosen such that twice the channel number was approximately the $\gamma$ ray energy. The Doppler shifting of the lines can be seen in this figure. The ${ }^{28} \mathrm{Si}$ peak in the ${ }^{17} \mathrm{O}$ spectrum is a good example. In all these spectra, only the $511 \mathrm{keV}$ peak and the $\mathrm{Ta}$ and $\mathrm{Au}$ Coulomb excitation lines are not shifted. As mentioned previously, these shifts caused some ambiguity in identifying the lines. Fortunately, there was at least one unique $\gamma$ ray for each exit channel in the ${ }^{16} \mathrm{O}+{ }^{16} \mathrm{O}$ and ${ }^{16} \mathrm{O}+{ }^{17} \mathrm{O}$ studies. In the ${ }^{16} \mathrm{O}+{ }^{18} \mathrm{O}$ reaction, there were many more exit channels (see figure 3 ) and we were unable to identify four of them. They were the channels resulting in the evaporation of $\mathrm{nt}, \alpha \mathrm{d}, \alpha \mathrm{t}$, or $\alpha^{3} \mathrm{He}$. Fortunately, these two-particle channels had large negative $Q$ values and a HAUSER*5 calculation indicated that these channels would contribute less than $0.1 \%$ to the total ${ }^{16} \mathrm{O}+{ }^{18} \mathrm{O}$ fusion yield.

The spectra in figure 5 show that the unique indicators of carbon buildup on the target are not visible within the statistical errors. For ${ }^{16,17} \mathrm{O}$ beams the unique signature of ${ }^{16,17} \mathrm{O}+{ }^{12} \mathrm{C}$ fusion is the $417 \mathrm{keV} \gamma$ ray from an ${ }^{26} \mathrm{Al}$ recoil. This is an open channel for ${ }^{16,17} \mathrm{O}+{ }^{12} \mathrm{C}$ but not for ${ }^{16} \mathrm{O}+{ }^{16} \mathrm{O}$ or ${ }^{16} \mathrm{O}+{ }^{17} \mathrm{O}$. There is a small peak in the ${ }^{16} \mathrm{O}+{ }^{17} \mathrm{O}$ spectrum at $1370 \mathrm{keV}$ which might be attributed to ${ }^{12} \mathrm{C}+{ }^{17} \mathrm{O}$ but since we do not see the ${ }^{26} \mathrm{Al}$ line nor any $\gamma$ rays from ${ }^{24} \mathrm{Na}$ (another unique signature of ${ }^{12} \mathrm{C}+{ }^{17} \mathrm{O}$ fusion) we assume that this line is due entirely to the ${ }^{24} \mathrm{Mg}+\alpha+\alpha+\mathrm{n}$ exit channel which has a large negative $Q$ value ( $-4.54 \mathrm{MeV}$ ).

Tests with a carbon target confirmed that the $417 \mathrm{keV}$ line from ${ }^{26} \mathrm{Al}$ was an indicator of the amount of carbon on the target. There were other unique channels to check for carbon buildup when running an ${ }^{17} \mathrm{O}$ or an ${ }^{18} \mathrm{O}$ beam. We were unable to detect any carbon on the targets during these experiments to a sensitivity of $<0.1 \mu \mathrm{g} / \mathrm{cm}^{2}$, and separate runs with a blank Ta target indicated that the carbon content of the $\mathrm{Ta}$ was also negligible. 

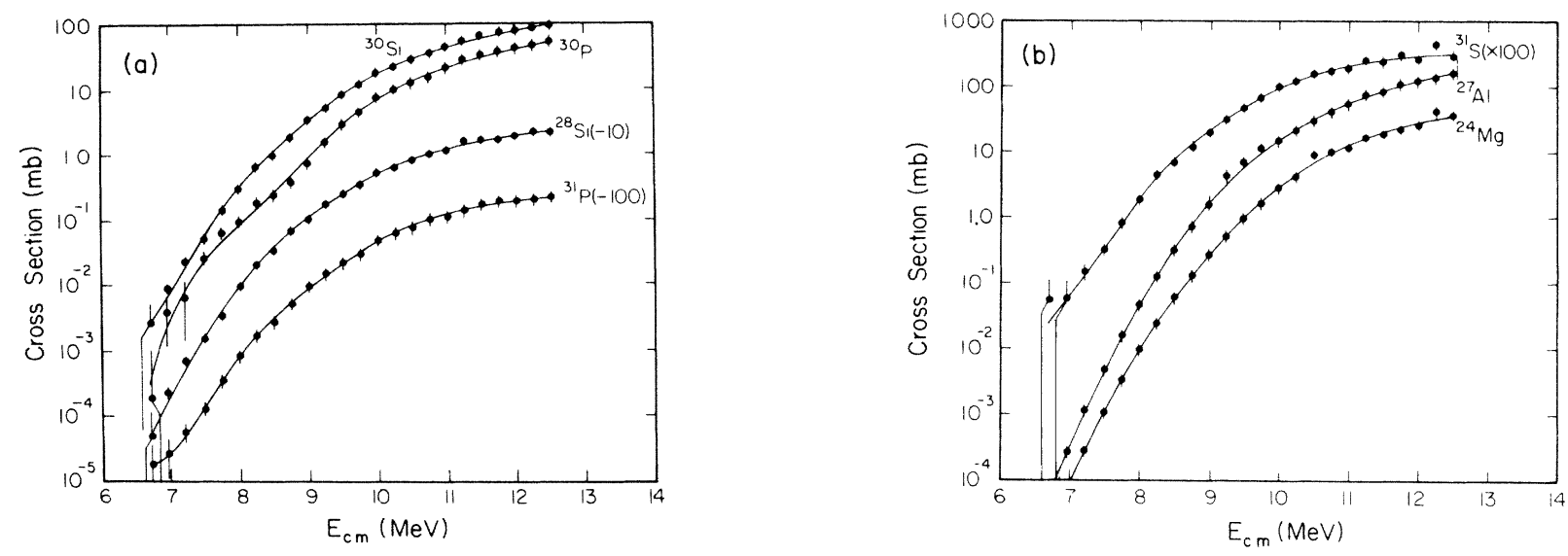

FIG. 6. The partial fusion reaction cross sections for the ${ }^{16} \mathrm{O}+{ }^{16} \mathrm{O}$ reaction.
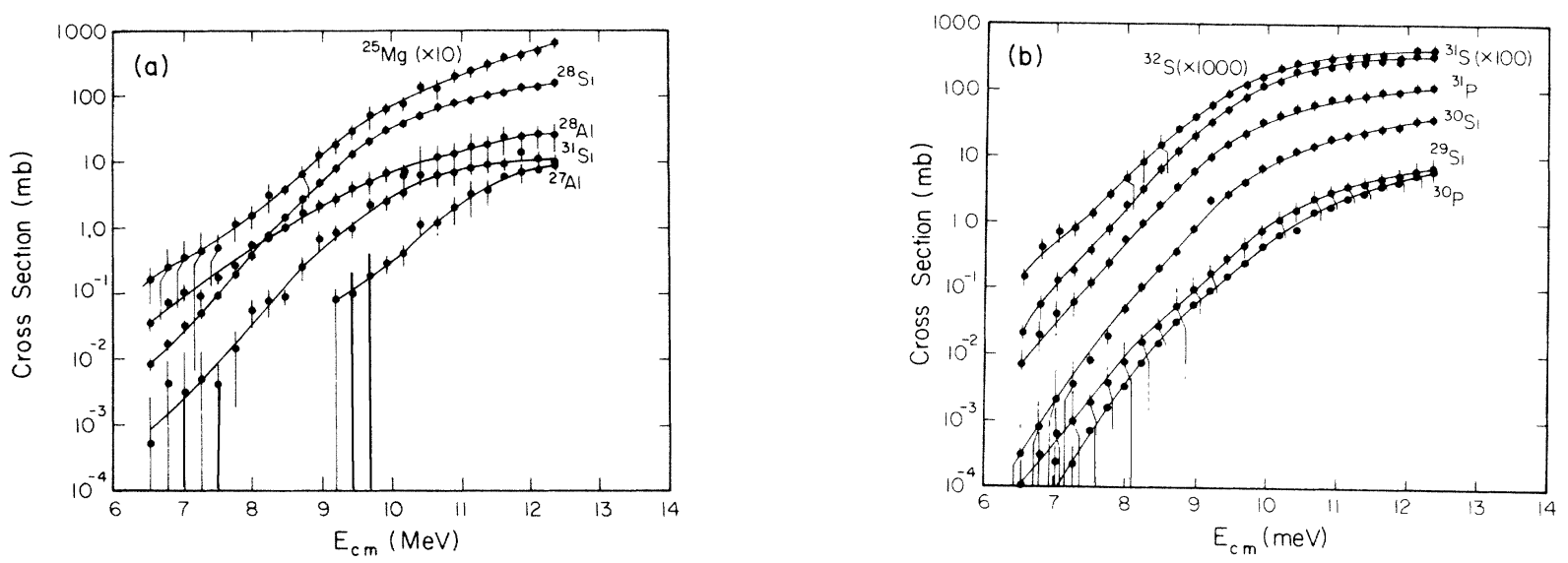

FIG. 7. The partial fusion reaction cross sections for the ${ }^{16} \mathrm{O}+{ }^{17} \mathrm{O}$ reaction.
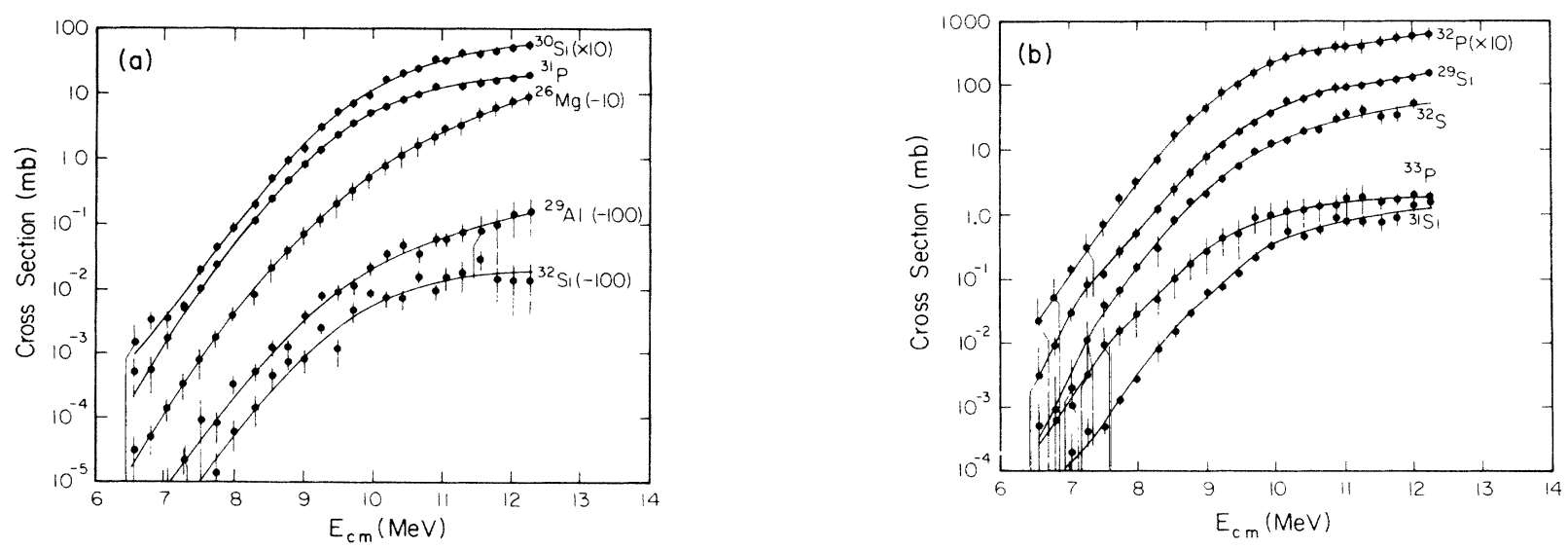

FIG. 8. The partial fusion reaction cross sections for the ${ }^{16} \mathrm{O}+{ }^{18} \mathrm{O}$ reaction. 


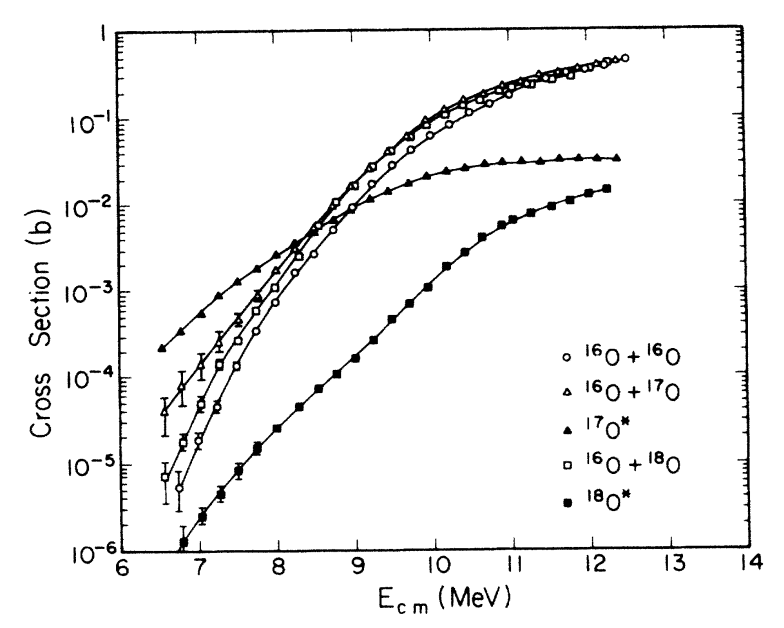

FIG. 9. The total fusion reaction cross section for ${ }^{16} \mathrm{O}+{ }^{16} \mathrm{O}$, ${ }^{16} \mathrm{O}+{ }^{17} \mathrm{O}$, and ${ }^{16} \mathrm{O}+{ }^{18} \mathrm{O}$. The inelastic cross sections for ${ }^{16} \mathrm{O}+{ }^{17} \mathrm{O}^{*}$ and ${ }^{16} \mathrm{O}+{ }^{18} \mathrm{O}^{*}$ are also shown.

The total fusion cross sections for ${ }^{16} \mathrm{O}+{ }^{16} \mathrm{O},{ }^{16} \mathrm{O}+{ }^{17} \mathrm{O}$, and ${ }^{16} \mathrm{O}+{ }^{18} \mathrm{O}$ are shown in figure 9 . The statistical errors on the data are $\pm 5 \%$ at the highest energies and \pm $20 \%$ to $\pm 50 \%$ at the lowest. These errors include the difficulty of estimating the background under a peak, especially at low energies. The systematic errors are dominated by the uncertainty in the target thickness and so are about $\pm 5 \%$.

The inelastic cross sections shown in figure 9 are derived from the yield of the $871 \mathrm{keV}$ and the $1982 \mathrm{keV} \gamma$ rays emitted by ${ }^{17} \mathrm{O}^{*}$ and ${ }^{18} \mathrm{O}^{*}$ respectively. Because only one $\gamma$ ray was observed, these cross sections are probably less reliable than the partial fusion cross sections, where more than one $\gamma$ ray was observed, and certainly less reliable than the total fusion cross sections. The systematic errors associated with the inelastic cross sections are estimated to be 3 or 4 times larger than the random errors.

We did not observe an ${ }^{17} \mathrm{O} \gamma$ ray during the ${ }^{16} \mathrm{O}+{ }^{18} \mathrm{O}$ experiment and so we conclude that the neutron transfer cross section was negligible.

\section{v. DISCUSSION}

Figure 10 shows the ${ }^{16} \mathrm{O}+{ }^{16} \mathrm{O}$ fusion cross section plotted as the astrophysical $S$ factor. The solid line in the figure is the result of an adiabatic time dependent Hartree-Fock calculation by Reinhard et al. ${ }^{21}$ They used a modified Bonche-Koonin-Negele interaction to describe the fusing nuclei. The agreement between the calculation and experiment is striking, especially since the calculation has not been normalized to the data. Using their calculation it is possible to extrapolate the data down into the astrophysically interesting region at $4 \mathrm{MeV}$ and find that the cross section there is $1.1 \times 10^{26} \mathrm{MeV} \mathrm{b}$ which is in good agreement with the calculations of Fowler, Caughlin, and Zimmerman ${ }^{22}$. Recent data reported by $\mathrm{Wu}$ and Barnes ${ }^{23}$ and Hulke et al. ${ }^{24}$ are also shown in the figure.

Our previous discussion of oxygen fusion ${ }^{25}$ led us to conclude that there was very little difference between the

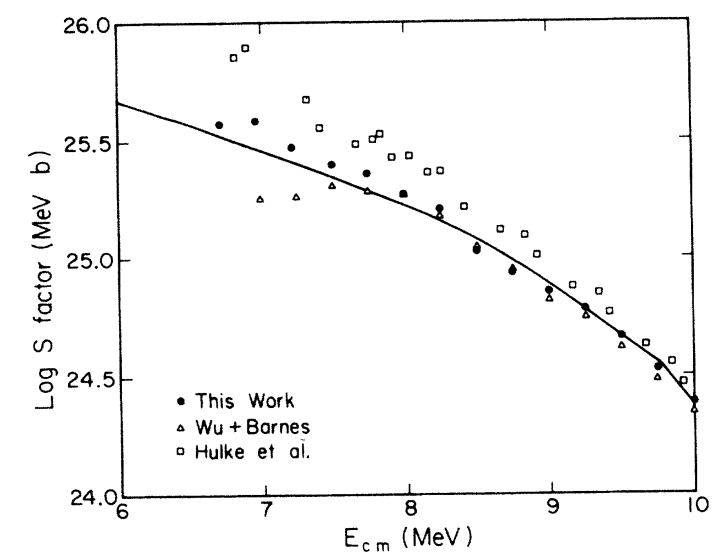

FIG. 10. The ${ }^{16} \mathrm{O}+{ }^{16} \mathrm{O}$ fusion cross section plotted as the astrophysical $S$ factor and compared to the adiabatic time dependent Hartree-Fock calculation of Reinhard et al. Recent data by $\mathrm{Wu}$ and Barnes, and Hulke et al. are also shown.

fusion cross sections for ${ }^{16} \mathrm{O}+{ }^{16} \mathrm{O}$ and ${ }^{16} \mathrm{O}+{ }^{18} \mathrm{O}$. We are now in a position to include the ${ }^{16} \mathrm{O}+{ }^{17} \mathrm{O}$ data in this comparison, and the results are interesting.

Figure 11 shows the effective one-dimensional potentials derived from our data for each of the oxygen systems. It is possible to scale the potentials according to the radii of the fusing systems and when this is done the ${ }^{16} \mathrm{O}$ and ${ }^{18} \mathrm{O}$ potentials are essentially the same $\mathrm{e}^{25}$. These potentials were derived using the inversion procedure of Balantekin et al. ${ }^{1}$ We smoothed the data in order to facilitate the analysis which required first and second derivatives. The amount of smoothing required was very minor. Two or three points from each cross section fell obviously above or below the general trend of the data. These data points were increased or decreased by no more than $1 \sigma$ (statistical) to bring them into better agreement with the trend of the data. The cross sections were also partially normalized to the $24 \mathrm{MeV}$ data point in ${ }^{16} \mathrm{O}+{ }^{16} \mathrm{O}$. By this we mean that the ${ }^{16} \mathrm{O}+{ }^{17} \mathrm{O}$ cross section was shifted

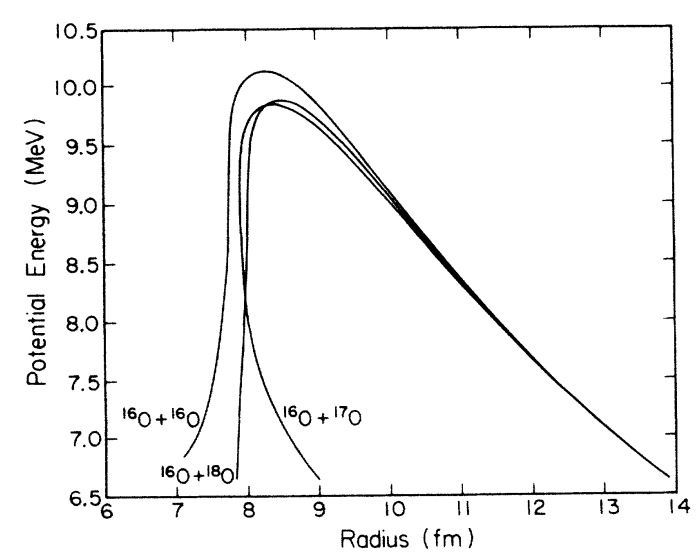

FIG. 11 The effective one-dimensional potential barrier for ${ }^{16} \mathrm{O}+{ }^{16,17,18} \mathrm{O}$ fusion using the inversion procedure of Ref. 1 . The unusual shape of the ${ }^{16} \mathrm{O}+{ }^{17} \mathrm{O}$ potential suggests that this system cannot be described by a one-dimensional potential. 


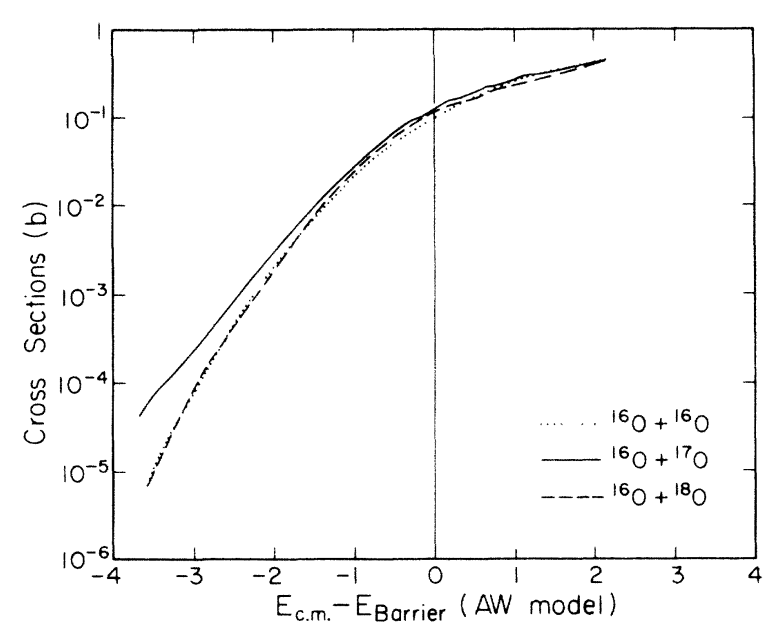

FIG. 12. The total fusion cross sections plotted as a function of energy below the barrier. The barrier top is defined as in Ref. 26.

down $1 \sigma$ (systematic) and the ${ }^{16} \mathrm{O}+{ }^{18} \mathrm{O}$ cross section was shifted up by $1 \sigma$, in order to bring the asymptotic behavior of the cross sections into better agreement above the barrier where the transmission coefficients for all three reactions are approaching 1.0.

Alternatively, it is possible to look directly at the cross sections as a function of the energy below the barrier $\left(E_{c . m}-E_{\text {barrier }}\right.$ ) (see figure 12). We have chosen to use the model of Akyuz and Winther ${ }^{26}$ to define the barrier top. It was derived from a global fit to elastic scattering data and predicts smooth trends for the change in barrier heights with mass. The unusual feature of figures 11 and 12 is that the ${ }^{17} \mathrm{O}$ data do not scale with the other systems and at low energies the one-dimensional potential barrier ceases to have any physical meaning. The strong backbend in the ${ }^{17} \mathrm{O}$ potential in figure 11 is similar to what was found for the $\mathrm{Ni}+\mathrm{Ni}$ systems analyzed by Balantekin et al. ${ }^{1}$ We feel that this is positive evidence for the breakdown of the one-dimensional model, and it is only fortuitous that the model has enough degrees of freedom to include the shapes of both the ${ }^{18} \mathrm{O}$ and the ${ }^{16} \mathrm{O}$ data. We will show below that a Woods-Saxon potential can describe the ${ }^{16} \mathrm{O}+{ }^{16} \mathrm{O}$ data very well but that a coupled channels calculation is necessary to describe the ${ }^{18} \mathrm{O}$ and ${ }^{17} \mathrm{O}$ data when they are constrained to the same WoodsSaxon potential.

We have used the two channel coupled channels approximation as presented by Landowne ${ }^{6}$. This model assumes constant coupling between the fusion channel and the inelastic channel, a somewhat unrealistic situation, but is easy to calculate and gives a qualitative understanding of the $Q$ value effect on sub-barrier fusion reactions. The assumption of constant coupling is not too unreasonable an approximation for oxygen fusion because the coupling to the inelastic channel is weak and the $Q$ values are relatively large. Also, it is appropriate to use a two channel model because the data indicate that the other channels, such as neutron transfer, do not contribute significantly to the total reaction cross section. It should be noted, though, that this model ignores all spins of the interacting nuclei. Thus, we cannot describe the inelastic cross sections accurately. We have not analyzed our data with the standard coupled channels codes because none are capable of handling the non-zero spin of the ${ }^{17} \mathrm{O}$ ground state.

The model of Landowne, Dasso, and Winther ${ }^{6,7}$ assumes that the form factor for each channel is a constant, $F$. Also, the effective potential must be the same in all channels. The coupled channel equations become:

$$
\begin{aligned}
& {\left[\frac{-\hbar^{2}}{2 \mu} \frac{d^{2}}{d x^{2}}+V(x)-E_{0}\right] \Phi_{0}=-F \Phi_{1},} \\
& {\left[\frac{-\hbar^{2}}{2 \mu} \frac{d^{2}}{d x^{2}}+V(x)-E_{1}\right] \Phi_{1}=-F \Phi_{0}+Q \Phi_{1}}
\end{aligned}
$$

where we have explicitly assumed that two channels participate in the reaction. These equations are diagonalizable with eigenvalues

$$
\lambda=\frac{-Q \pm\left(Q^{2}+4 F^{2}\right)^{1 / 2}}{2} .
$$

It is then necessary to solve two one-dimensional optical model equations with energy shifts $E \pm \lambda$. The resulting wave functions, or transmission coefficients, can be rotated back to the physical solutions by using the eigenvectors of the coupled equations.

We have incorporated this trick into a one-dimensional incoming wave boundary condition (IWBC) model $^{27}$ and into a WKB barrier penetration code which solves for the transmission coefficients in an arbitrary potential. The two channel coupled channels calculation was then approximated by combining the transmission coefficients with the appropriate weights to calculate the total fusion cross section.

We will only discuss the results of the IWBC calculations. The WKB calculations give similar results; we attribute the small differences to the fact that the WKB approximation is only useful below the barrier top. The IWBC is of course valid at all energies and is also useful for calculating elastic scattering cross sections.

Figure 13 shows the IWBC fit to the ${ }^{16} \mathrm{O}+{ }^{16} \mathrm{O}$ data

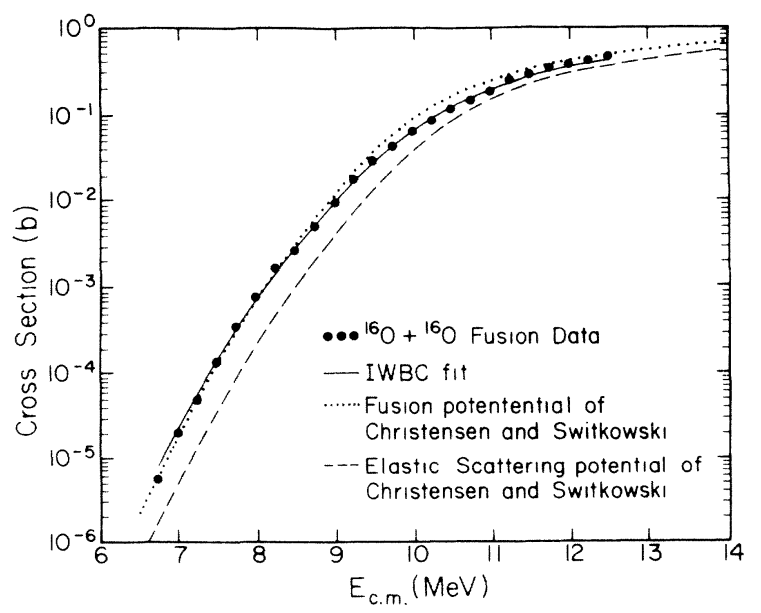

FIG. 13. An IWBC fit to the ${ }^{16} \mathrm{O}+{ }^{16} \mathrm{O}$ fusion data. The model used a simple, real, Woods-Saxon potential: $V_{0}=50$ $\mathrm{MeV}, r_{0}=1.29 \mathrm{fm}$, and $a=0.460 \mathrm{fm}$. The $\chi^{2}$ of the fit was 1.66 per degree of freedom. The fit of Christensen and Switkowski is also shown. (See text.) 
with the inelastic coupling form factor set to 0 . The potential was constrained to a Woods-Saxon form and we found that the well depth was not an important parameter. Equally small $\chi^{2}$ values could be found with the depth set to $50 \mathrm{MeV}$ or $90 \mathrm{MeV}$ and so we fixed it at 50 $\mathrm{MeV}$ for all of the analyses. The fit shown in figure 13 was chosen from amongst all possible values in a twodimensional space. The reduced radius was $1.29 \mathrm{fm}$ and the diffuseness $0.460 \mathrm{fm}$. The $\chi^{2}$ of this fit was 1.66 . Our first important conclusion is that these are very reasonable values. Previously, Christensen and Switkowski ${ }^{28}$ had attempted to fit both the sub-barrier fusion cross section and the ${ }^{16} \mathrm{O}$ elastic scattering cross sections of Spinka and Winkler ${ }^{8}$ using the same potential and did not succeed. They published a best fit to each set of data and their results are shown in figures 13 and 16 . Using our new parameters, we find that we can achieve an excellent fit to our fusion data and simultaneously to the elastic scattering data of Spinka and Winkler. The Christensen elastic potential does a better job of fitting the high energy part of the elastic data but does a poorer job near the barrier. Neither fit is satisfactory in a $\chi^{2}$ sense but both are remarkably good for such a simple model. Our ability to fit both fusion and elastic cross sections indicates that there is no longer any ambiguity in the description of ${ }^{16} \mathrm{O}$ fusion and indicates that the fusion process probably contains no undiscovered mechanisms.

Our next step was to take the ${ }^{16} \mathrm{O}$ potential, scale it for different masses, and use it to describe the fusion of ${ }^{16} \mathrm{O}+{ }^{18} \mathrm{O}$. The only parameter in this fit was the magnitude of the coupling to the first excited state in ${ }^{18} \mathrm{O}$ at $1.98 \mathrm{MeV}$. With no coupling, the $\chi^{2}$ of the fit is 11.7 , but with a coupling strength of $0.48 \mathrm{MeV}$ the $\chi^{2}$ drops to 3.0. The best fit allowing the Woods-Saxon potential parameters to change is not significantly better than this, 2.7 , because the dominant contribution to the $\chi^{2}$ comes between

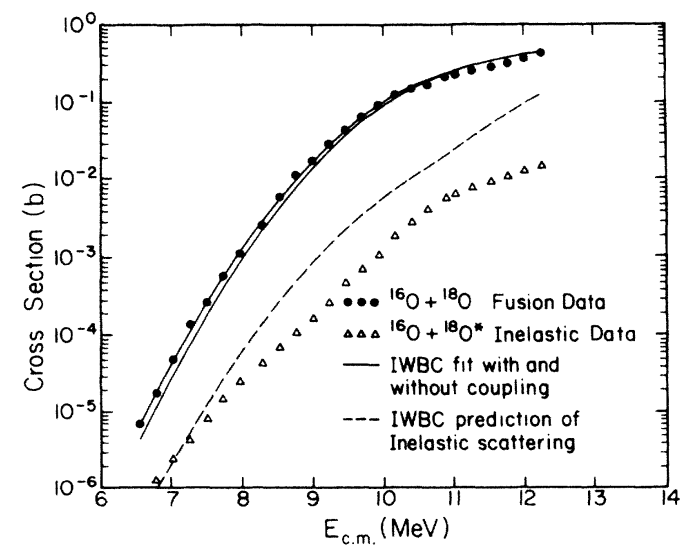

FIG. 14. An IWBC fit to the ${ }^{16} \mathrm{O}+{ }^{18} \mathrm{O}$ fusion data. The ${ }^{16} \mathrm{O}+{ }^{16} \mathrm{O}$ potential was used but a constant coupling of 0.48 $\mathrm{MeV}$ to the first excited state in ${ }^{18} \mathrm{O}$ was included. The coupling strength form factor was the only parameter in this fit. The model prediction without coupling is also shown. The inelastic data and the model prediction of the inelastic cross section are shown in the lower portion of the figure.

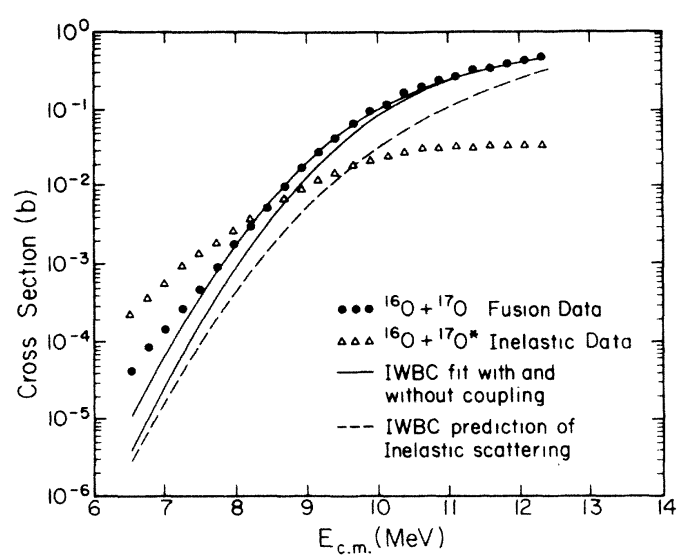

FIG. 15. An IWBC fit to the ${ }^{16} \mathrm{O}+{ }^{17} \mathrm{O}$ fusion data. The ${ }^{16} \mathrm{O}+{ }^{16} \mathrm{O}$ potential was used but a constant coupling of 0.60 $\mathrm{MeV}$ to the first excited state in ${ }^{17} \mathrm{O}$ was included. The coupling strength form factor was the only parameter in this fit. The model prediction without coupling is also shown. The inelastic data and the model prediction of the inelastic cross section are shown in the lower portion of the figure.

11 and $12 \mathrm{MeV}$ where two data sets meet. (The ${ }^{16} \mathrm{O}+$ ${ }^{18} \mathrm{O}$ data were collected in two separate runs with different targets and different experimental conditions. We took no steps to normalize these data and so the slight variation is the result of two different systematic errors.) We feel that the one parameter fit, with coupling, is very good. The calculated cross sections are shown in figure 14. For completeness we have also calculated the inelastic cross section using the parameters that describe the fusion cross section. We would not expect this calculation to fit the data since the assumption of constant coupling is not realistic, yet the magnitude and shape of the calculated cross

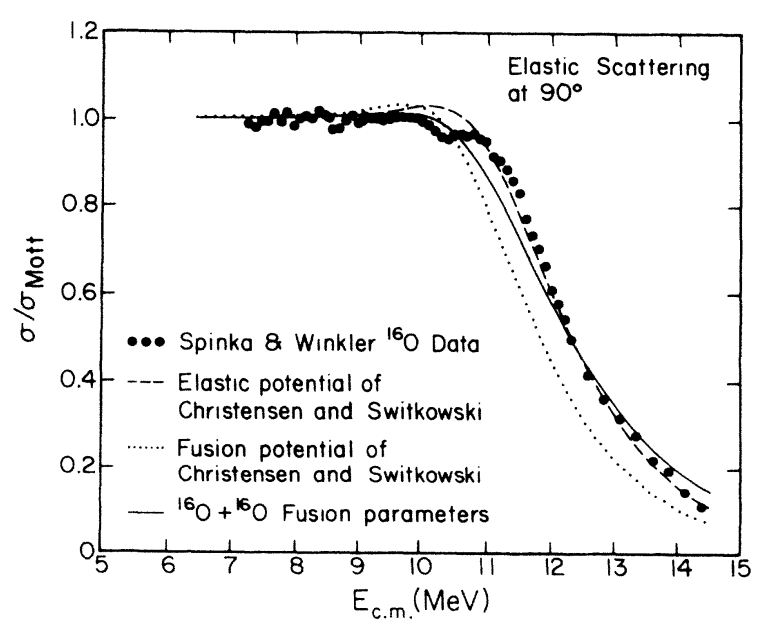

FIG. 16. The elastic scattering excitation function for ${ }^{16} \mathrm{O}+{ }^{16} \mathrm{O}$ collected at 90 degrees. The best fit of Ref. 28 is compared to their best fusion potential and the potential used in this work to describe both processes. 
section suggests that a more accurate description of the inelastic channel might completely describe the fusion and inelastic data.

Finally, we applied our model to the ${ }^{17} \mathrm{O}$ data. Here, the channel coupling is very important because the first excited state of ${ }^{17} \mathrm{O}$ is at $0.871 \mathrm{MeV}$. We used the ${ }^{16} \mathrm{O}$ potential and allowed the inelastic coupling constant to vary. The best fit is shown in figure 15 . It was achieved with a coupling constant of $0.60 \mathrm{MeV}$ and the $\chi^{2}$ of the fit drops from 23 to 3.0. These data were taken in one run and so the structure at low energy in the ${ }^{17} \mathrm{O}$ fusion cross section is unexplained. It may be that the target was contaminated with carbon and so we are seeing $\mathrm{C}+\mathrm{O}$ fusion at some low level, but as we mentioned before there were no other indications of carbon on our target. Therefore, it seems that the low energy structure is real although the reader should be reminded that the error bars are very large for the low energy data (see figure 9).

The calculated inelastic cross section for ${ }^{16} \mathrm{O}+{ }^{17} \mathrm{O}$ does not represent the data at all. Neither the shape nor the magnitude is correct, but it is obvious that the assumption of constant coupling is not very useful in a situation where the $Q$ value is small and the coupling constant is large. (Although, one might conjecture that overpredicting the inelastic cross section above the barrier and underpredicting it below the barrier is the result of an additional channel coupling. This type of behavior has been discussed by Dasso et al. ${ }^{7}$ and would be consistent with the low energy structure seen in the ${ }^{16} \mathrm{O}+{ }^{17} \mathrm{O}$ fusion data.)

\section{CONCLUSION}

We have presented our measurements of the fusion cross sections for the ${ }^{16} \mathrm{O}+{ }^{16} \mathrm{O},{ }^{16} \mathrm{O}+{ }^{17} \mathrm{O}$, and ${ }^{16} \mathrm{O}+{ }^{18} \mathrm{O}$ systems. The data indicate that only inelastic excitations contribute to the total reaction cross section at energies below the barrier and therefore a simple two channel coupled channels calculation fits the data. This suggests to us that a coupled channels approach to sub-barrier fusion is the appropriate way to describe the sub-barrier enhancements that are seen in heavier systems.

We do not find any discrepancy between the potentials required to describe the fusion and elastic scattering of the oxygen isotopes. A more complete (microscopic) calculation of the fusion, inelastic, and elastic cross sections is underway at Caltech.

\section{ACKNOWLEDGMENTS}

This work was supported in part by grants from the National Science Foundation (No. PHYS82-15500) and the Department of Energy (No. DEAC-0381-ER40050). We are grateful for the support of one of us (J.T.) by a Millikan Fellowship and we would like to acknowledge useful discussions with C.A. Barnes, S.E. Koonin, and A.B. Balantekin.
*Permanent address: Australian National University, Canberra, Australia.

1B. Balantekin, S. Koonin, and J. Negele, Phys. Rev. C 28, 1565 (1983).

${ }^{2}$ M. Inui and S. Koonin, Phys. Rev. C 30, 175 (1984).

3J. Blocki et al., Ann. Phys. (N.Y.) 105, 427 (1977).

${ }^{4}$ M. Beckerman et al., Phys. Rev. Lett. 45, 1472 (1980).

${ }^{5}$ M. Beckerman et al., Phys. Rev. C 25, 837 (1982).

${ }^{6} \mathrm{~S}$. Landowne, in Proceedings of the International Conference on Fusion Reactions Below the Coulomb Barrier, Cambridge 1984, edited by S. Steadman (Springer-Verlag, Berlin, 1985).

${ }^{7}$ C.H. Dasso, S. Landowne, and A. Winther, Nucl. Phys. A405, 381 (1983).

${ }^{8}$ H. Spinka and H. Winkler, Nucl. Phys. A233, 456 (1974).

${ }^{9}$ Monsanto Research Co., Mound Laboratory, Miamisburg, Ohio.

10P.R. Christensen, Z.E. Switkowski, and R.A. Dayras, Nucl. Phys. A280, 189 (1977).

${ }^{11}$ National Bureau of Standards Report No. SRM-4275-164, 1981.

12J.P.S. Pringle, J. Electrochem. Soc. 119, 482 (1972).

${ }^{13}$ D. Phillips and J.P.S. Pringle, Nucl. Instrum. Methods 135, 389 (1976).

${ }^{14} \mathrm{M}$. Mendenhall, private communication.

${ }^{15}$ L.C. Northcliffe and R.F. Schilling, Nucl. Data Tables A7,
233 (1970).

16P.E. Hodgson, Nuclear Reactions and Nuclear Structure (Clarendon, Oxford, 1971).

${ }^{17}$ F.M. Mann, Hanford Engineering and Development Laboratory, Report No. HEDL-TME 78-83, 1978 (unpublished).

18P.M. Endt and C. Van DerLeun, Nucl. Phys. A214, 1 (1973).

${ }^{19}$ A. Gilbert and A.G.W. Cameron, Can. J. Phys. 43, 1446 (1965).

${ }^{20}$ K. Debertin and U. Schotzig, Nucl. Instrum. Methods 158, 471 (1979).

${ }^{21}$ P.G. Reinhard et al., Phys. Rev. C 30, 878 (1984).

${ }^{22}$ W.A. Fowler, C.R. Caughlin, and B.A. Zimmerman, Annu. Rev. Astron. Astrophys. 13, 69 (1975).

${ }^{23}$ C.S. Wu and C.A. Barnes, Nucl. Phys. A422, 373 (1984).

${ }^{24}$ G. Hulke, C. Rolfs, and H.P. Trautvetter, Z. Phys. A 297, 161 (1980).

25J. Thomas et al., Phys. Rev. C 31, 1980 (1985).

260. Akyuz and A. Winther, in Proceedings of the Enrico Fermi International School of Physics, 1979, edited by R.A. Broglia, C.H. Dasso, and R.A. Ricci (North-Holland, Amsterdam, 1980), p. 492.

${ }^{27}$ G.H. Rawitscher, Nucl. Phys. 85, 337 (1963).

${ }^{28}$ P.R. Christensen and Z.E. Switkowski, Nucl. Phys. A280, 205 (1977). 